\title{
New Sesame Equation of State for Tantalum
}

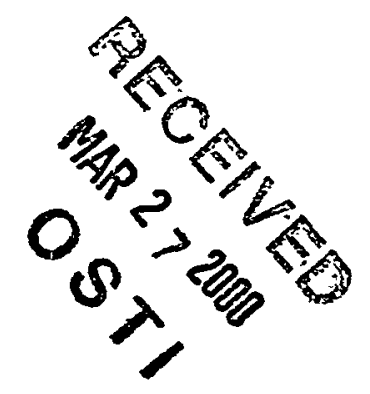




\section{An Affirmative Action/Equal Opportunity Employer}

This report was prepared as an account of work sponsored by an agency of the United States Government. Neither The Regents of the University of California, the United States Government nor any agency thereof, nor any of their employees, makes any warranty, express or implied, or assumes any legal liability or responsibility for the accuracy, completeness, or usefulness of any information, apparatus, product, or process disclosed, or represents that its use would not infringe privately owned rights. Reference herein to any specific commercial product, process, or service by trade name, trademark, manufacturer, or otherwise, does not necessarily constitute or imply its endorsement, recommendation, or favoring by The Regents of the University of Califormia, the United States Government, or any agency thereof. The viezs and opinions of authors expressed herein do not necessarily state or reflect those of The Regents of the University of California, the United States Government, or any agency thereof. Los Alamos National Laboratory strongly supports academic freedom and a researcher's right to publish; as an institution, howeoer, the Laboratory does not endorse the viewpoint of a publication or guarantee its technical correctness. 


\section{DISCLAIMER}

Portions of this document may be illegible in electronic image products. Images are produced from the best available original document. 
New Sesame Equation of State for Tantalum

C. W. Greeff

J. D. Johnson 


\title{
New Sesame Equation of State for Tantalum
}

by

C. W. Greeff and J. D. Johnson

\begin{abstract}
A new Sesame equation of state (EOS) table has been created for tantalum. This EOS incorporates new high pressure Hugoniot data and diamond anvil cell compression data. The new EOS gives better agreement with this data as well as with sound speeds and Hugoniot curves of porous samples.
\end{abstract}

We have created a new Sesame table, material number 3522, for the equation of state (EOS) of tantalum (Ta). The previous Ta EOS is material number 3520. Since it was developed, significant EOS data have been published, including high pressure Hugoniot data [1], Hugoniot data on porous samples [2], sound speed data on shocked Ta [3], and diamond anvil cell (DAC) room temperature compression data [4]. This new EOS has been developed in response to these data.

We find that in order to match simultaneously the Hugoniot and DAC data, an unconventional volume dependence of the Gruneisen parameter $\gamma$ is required. These data are used to fix the model. Off-Hugoniot information from the sound speed and porous sample data is then used to test the new EOS. In all cases we find improved agreement with the data.

The present models are improved over the earlier ones by including melting in the nuclear model [5]. The new Sesame data also include melt and shear modulus tables.

Figure 1 shows the Hugoniot and room $T$ isotherm of $\mathrm{Ta}$ in the pressurevolume plane. Also shown are the results from the present model (3522) along with those from the earlier 3520 . At the time that 3520 was developed, 


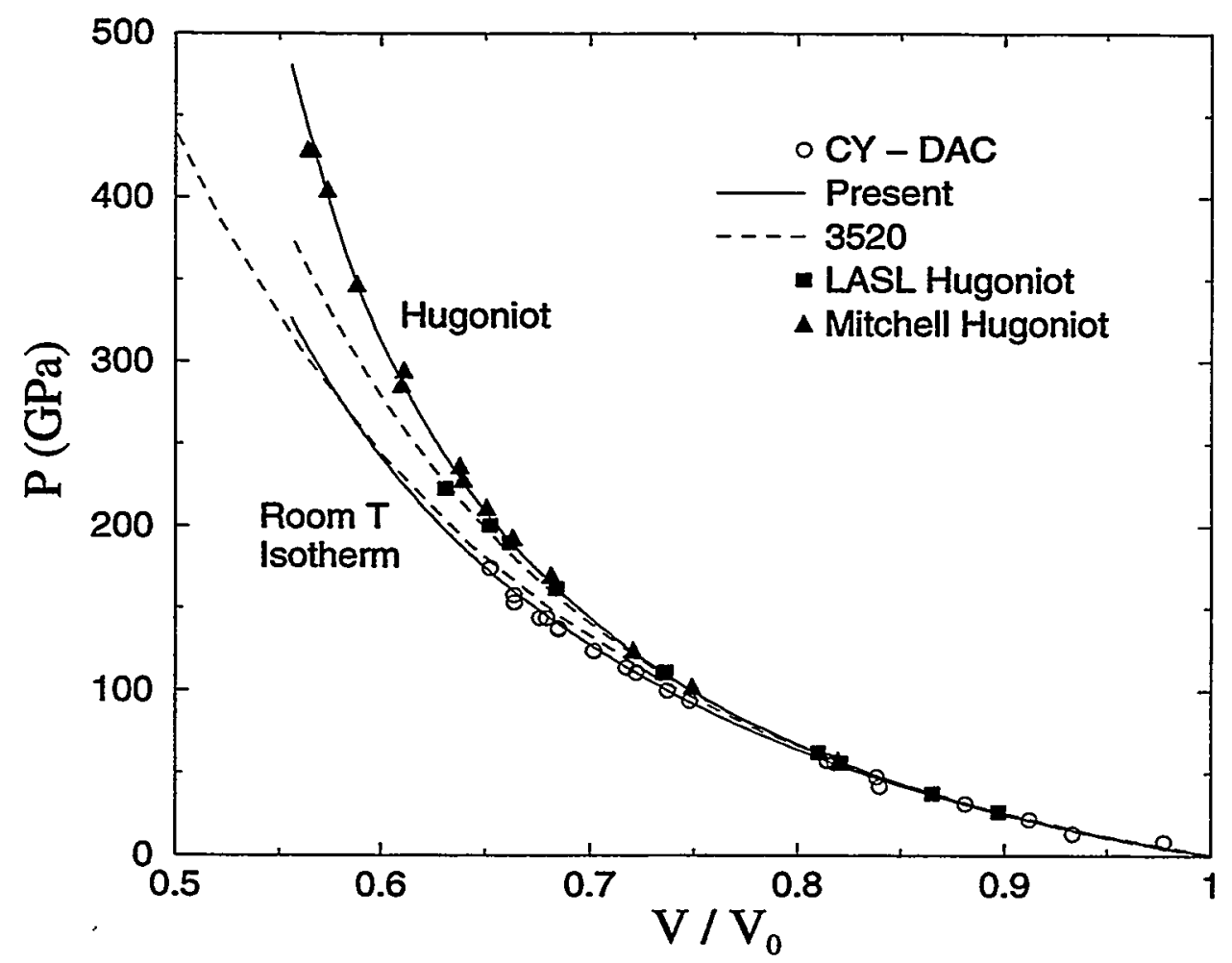

Figure 1: Hugoniot and room temperature isotherm of Ta. Open circles are the DAC room $T$ compression data of Cynn and Yoo [4]. Filled squares are the LASL principal Hugoniot data [6]; filled triangles are Hugoniot from Mitchell and Nellis [1]. Solid curves are the present model, Sesame 3522, and dashed curves are the older Sesame 3520.

neither the high-pressure Hugoniot data of Mitchell and Nellis [1], nor the room temperature DAC data of Cynn and Yoo. [4] were available. The linear $u_{s}\left(u_{p}\right)$ fit to the LASL data [6] was used as the basis for 3520 , which resulted in the Hugoniot being substantially too low at higher compressions. It is now thought that the highest point in the LASL data was corrupted by overtake of the shock by a release from the flyer back surface. ${ }^{1}$ Inclusion of this point in the fit resulted in a low extrapolation to higher compression.

The Gruneisen parameter $\gamma=V(\partial P / \partial E)_{V}$ is an important component

${ }^{1}$ This interpretation is due to J. N. Fritz. 


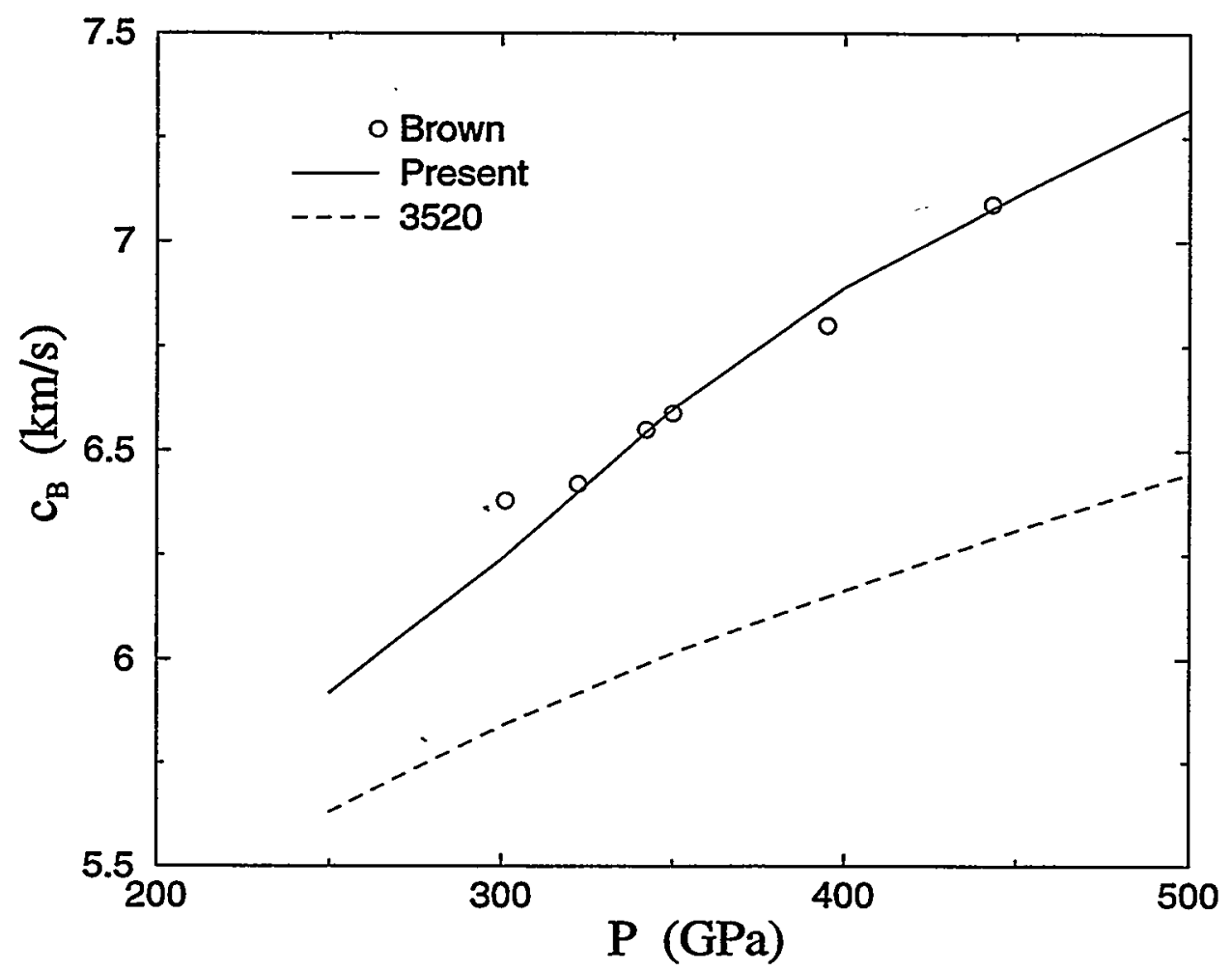

Figure 2: Sound speed in shocked Ta. Open circles are data of Brown and Shaner [3]. Solid curve is the present EOS, 3522. Dashed curve is EOS 3520.

of the modeling. The value of $\gamma$ at ambient conditions is well established by thermodynamic measurements as $\gamma=1.59$. Standard modeling [7] assumes that $\gamma$ initially decreases with increasing density and then approaches an asymptote $\gamma_{\infty}=2 / 3$. We find that the use of such a model in the present case results in the room temperature isotherm and the Hugoniot being too close together. This behavior is exhibited by 3520 in Figure 1. To match the data requires that $\gamma$ remain essentially constant over the range of compressions for which there is data.

To check the correctness of this modeling, it is desirable to compare the EOS to data that was not directly fitted. The bulk sound speed has been measured by Brown and Shaner [3] and provides an important check on the EOS. Figure 2 shows the computed sound speed from the new EOS, along 


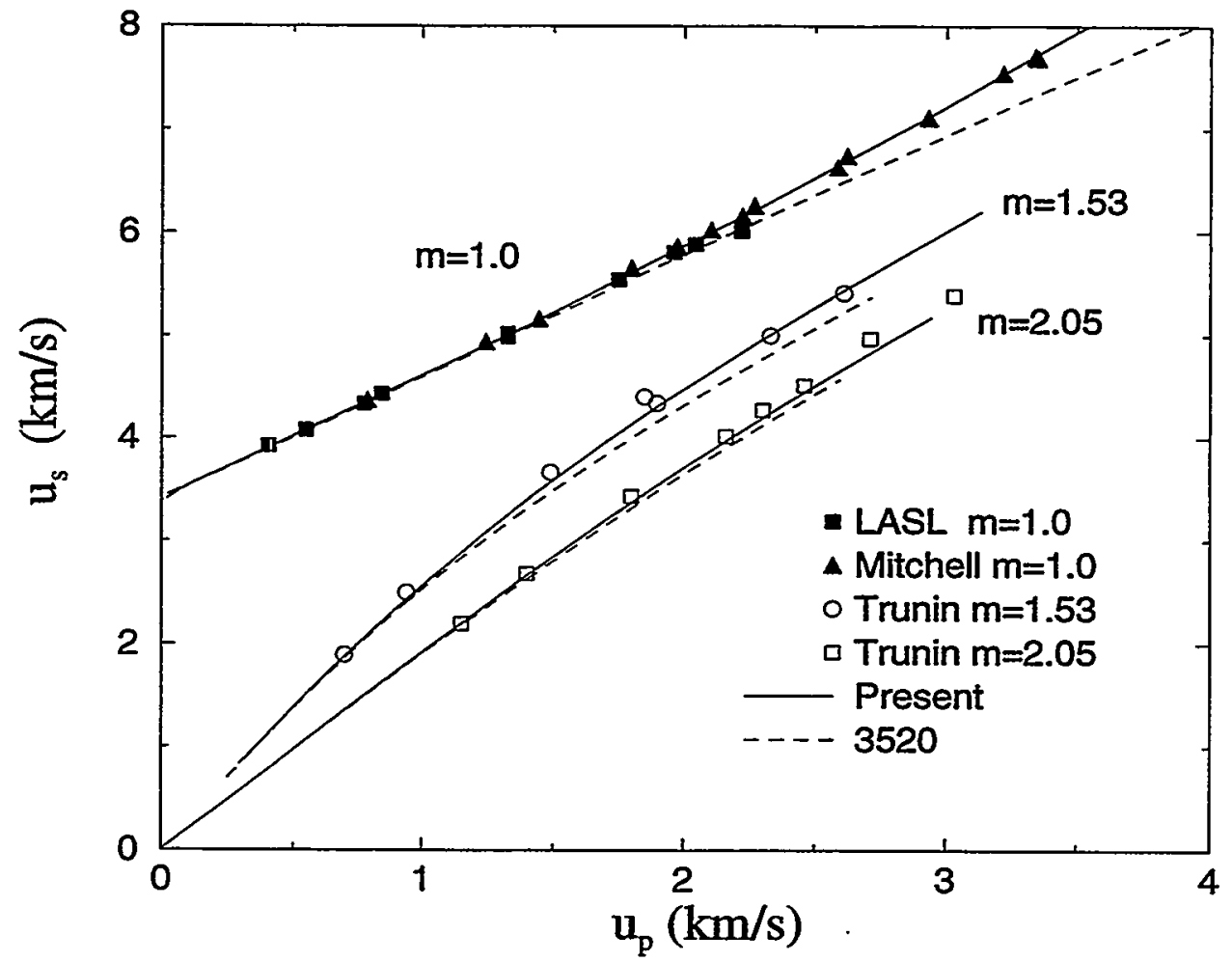

Figure 3: Hugoniots of porous Ta samples. Porosity is indicated by $m=$ $\rho_{\text {ref }} / \rho_{0}$, where $\rho_{\text {ref }}$ is the crystal density. Filled squares and filled triangles are the full density data from LASL [6] and Mitchell and Nellis [1], respectively. Open symbols are porous sample measurements from Trunin et al. [2]. Solid curves are calculated from the present EOS 3522, and dashed curves are from 3520 .

with that from EOS 3520, in comparison with the Brown and Shaner data. The new EOS clearly gives improved results.

Trunin et al. [2] have measured the Hugoniots of porous Ta samples, providing another set of data off the principal Hugoniot with which to test the EOS. A comparison of the present EOS, 3520, and their data is shown in Figure 3. The calculations assume that the crush-up of the pores is overdriven, so that the initial density is the only relevant parameter relating to the porosity. The new EOS agrees well with their data and is somewhat of 
an improvement over 3520 .

A short discussion is merited concerning the gas-liquid critical temperature $T_{c}$. We used a rule of thumb that the critical temperature in Kelvin is equal to the cohesive energy in $\mathrm{kcal} / \mathrm{mole}$ times 100 . The corresponding estimate is $T_{c}=18700 \mathrm{~K}$ for Ta. This method works quite well for the existing experimental $T_{c}$ data. However, the existing $T_{c}$ data set does not have temperatures high enough that the electrons would contribute to the EOS. Thus we first produced an EOS with no electronic contribution and adjusted that partial EOS to fit the estimated $T_{c}$. The $T_{c}$ from the total EOS, after including the electronic contribution, was lowered to $13400 \mathrm{~K}$. Other critical point parameters are $\rho_{c}=5.4 \mathrm{~g} / \mathrm{cm}^{3}$ and $P_{c}=1.6 \mathrm{GPa}$.

The threshold for shock melting was established by Brown and Shaner to be $\approx 300 \mathrm{GPa}$ [3]. The present calculations incorporate a melting model. Unfortunately the calculated threshold for shock melting is nearly $400 \mathrm{GPa}$. The reason for this discrepancy is not well understood. The melting criterion used in these calculations is a simple Lindemann rule. The temperature at shock melting is $\approx 1 \mathrm{eV}$. This leads to the possibility of large electronic contributions to the free energy and of complicated interactions between electronic and nuclear excitations. Such complications are beyond the scope

of the present modeling, and so the correct physics behind the shock melting threshold is left as an open question.

In conclusion, we have created a new Sesame EOS table for $\mathrm{Ta}$, which incorporates new experimental information. The modified modeling required to fit the principal Hugoniot and room temperature isotherm has been tested against off-Hugoniot measurements and found to improve agreement with data. The new EOS needs to be tested in hydrocode calculations. Barring any unforeseen difficulties, the new EOS, Sesame 3522, is to be preferred over the preceding one, Sesame 3520.

\section{References}

[1] A. C. Mitchell and W. J. Nellis, J. Appl. Phys. 52, 3363 (1981).

[2] R. F. Trunin, G. V. Simakov, Yu. N. Sutulov, A. B. Medvedev, B. D. Rogozkin, and Yu. E. Federov, Sov. Phys.-JETP 69, 580 (1989). 
[3] J. M. Brown and J. W. Shaner, in Shock Waves in Condensed Matter1983 edited by J. R. Assay, R. A. Graham, and G. K. Straub (North Holland, Amsterdam 1984).

[4] H. Cynn and C.-S. Yoo, Phys. Rev. B 59, 8526 (1999).

[5] J. D. Johnson, High Pressure Research 6, 277 (1991).

[6] S. P. Marsh, LASL Shock Hugoniot Data (University of California Press, Berkeley 1980).

[7] A. V. Bushman, G. I. Kanel, A. L. Ni, and V. E. Fortov, Intense Dynamic Loading of Condensed Matter (Taylor and Francis, Washington 1993). 